\title{
PHYSICAL FITNESS OF PARAMEDIC STUDENTS DURING VOCATIONAL TRAINING - A FOLLOW-UP STUDY
}

Heikki Paakkonen $\mathrm{PhD}, \mathrm{RN}^{1^{*}}$, Joachim Ring Master of Health Care, $\mathrm{PT}^{1}$, Jyrki Kettunen PhD, Title of Docent, $\mathrm{PT}^{1}$

1 Arcada University of Applied Sciences, Helsinki, Finland.

*heikki.paakkonen@arcada.fi

\section{Abstract}

\section{Background}

Paramedics' work includes periods of physically light activity, but also involves short periods of relatively intense physical activity. Even during their studies paramedic students' days involve a lot of sitting, i.e. physically sedentary behaviour. We initiated a programme to motivate paramedic students to be physically active during their studies. In this study we report physical fitness among paramedic students who for one year participated in brief physical activity counselling consisting of both verbal and a written individually tailored exercise program

\section{Methods}

A total of 40 paramedic students (26 female, 14 male) had participated voluntarily in a physical activity programme alongside their studies. Subjects' maximal oxygen consumption and muscle strength were measured at baseline and at one-year follow-up. According to the results of the baseline tests all students were given an individual exercise programme, which included aerobic and muscle strength training.

\section{OPEN ACCESS ARTICLE}

Recommended Citation: Paakkonen H, Ring J and Kettunen J. Physical fitness of paramedic students during vocational training - a follow-up study. Irish Journal of Paramedicine. 2018. 3(1).

Received: 05 April 2017

Accepted: 31 January 2018

Published: 20 February 2018

Copyright: $\odot$ 2018, the authors. This is an Open Access article distributed under the terms of the Creative Commons Attribution-Non-CommercialShare Alike 4.0 International licence which permits use, distribution, and reproduction in any medium, provided the original work and any attributes thereof are properly cited, are distributed under the same licence, and that the work is not used for commercial purposes.

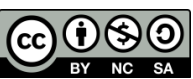

Funding: No funding declared.

Competing interests: The authors have declared that no competing interests exist.

Provenance and review: Not commissioned, peer-reviewed.

\section{Results}

Sixty-five percent (26/40) of the students participated in the follow-up examinations. At baseline male students had higher mean maximal oxygen consumption (VO2Max) and, as expected, greater absolute muscle strength than their female counterparts (all p-values <0.05). During the follow-up females had improved their VO2Max on average by $2.4 \mathrm{ml} / \mathrm{kg} / \mathrm{min}$. Furthermore, females on average improved the number of squats by 4.2 [95\% Confidence Interval $(\mathrm{Cl})$ 0.01 to 8.33] repetitions, and males improved the number of push-ups (mean improvement $4.8,95 \% \mathrm{Cl} 1.93$ to 7.57 ), and left arm grip strength (mean improvement $4.0 \mathrm{~kg}, 95 \% \mathrm{Cl} 1.39$ to 6.53$)$.

\section{Conclusions}

It seems to be possible to maintain, and even slightly improve paramedic students' physical fitness with a self-directed, guided, home exercise programme. This approach could enhance prospective paramedics' willingness to develop the necessary physical qualities long-term throughout their clinical careers.

Keywords: paramedics, physical fitness, physical endurance, employee health, follow-up studies 


\section{Introduction}

Paramedics are health care workers who respond to all kinds of emergencies. Time pressure, the requirement to provide medical care in life-and-death situations and in often unfavourable conditions under the scrutiny of bystanders and relatives are characteristic of these paramedics' work.(1) Musculoskeletal injuries are reportedly especially common among emergency medical services (EMS) personnel. These injuries were typically sprains, strains and tears, and anatomically the back was the part of the body most often injured.(2-4) Furthermore, paramedics working in ambulances are reportedly prone to musculoskeletal disorders leading to sick leave and early retirement.(5-7)

Crill and Hostler showed (8) that in spite of the physical nature of paramedics' profession, EMS providers were significantly overweight and likely to lack sufficient back strength and flexibility to perform their tasks safely. The researchers concluded that this group of professionals could be at risk for occupational injury and targeted interventions were urgently recommended.

Students in general have been found to have at least equivalent health risk factor, such as obesity, to those in general population.(9) Nurses seem to be a high-risk population for back pain. In a study, conducted in Oceania with a population of nurses and midwives $(\mathrm{N}=7633), 88.4 \%$ of the participants had experienced neck, upper back or lower back problems, and $26.3 \%$ had experienced problems in all three body regions.(10) It seems reasonable to assume that these findings, albeit made with nursing students, could also occur among paramedic students. Physical activity habits established during vocational education may be of help to keep paramedics-to-be and nurses physically equal to their future physically demanding roles.(9)

Pre-employment physical capacity testing is widely used as a part of the recruitment process of prospective paramedics, indicating the importance of physical fitness for their physical work capacity. The work of paramedics may involve long periods of sedentary behaviour, such as sitting. However, it also involves short periods of relatively intense activity, often above the anaerobic threshold $(6,11)$, and the authors pointed out the need for high standards of physical fitness for ambulance personnel.

In Finland paramedics are trained at eight universities of applied sciences (UAS). At the recruitment stage it is not mandatory to test the applicants' physical fitness. Despite evidence indicating the need for adequate physical fitness (6) currently only two UASs continue to conduct pre-enrolment testing of applicants' physical capacity.

Arcada UAS is one of the two UASs in Finland to include such tests in the recruitment process. Paramedic students spent long periods sitting during their days studying. To avoid the adverse events of sedentary behaviour, the paramedic students in Arcada UAS were offered an opportunity to participate in a physical activity programme alongside their studies. The aim of this study is to report the physical fitness of paramedic students who participated in an individually tailored home exercise programme during one-year period.

\section{Materials and methods}

As a part of their studies, paramedic students in Arcada UAS in Helsinki Finland have the option to participate in a physical activity programme. The programme includes physical capacity tests in the second semester i.e. at baseline, and follow-up tests one year thereafter. According to the results of the baseline tests, all students were given an individual exercise programme, which included aerobic and muscle strength training. The exercise programme for each individual focused on those results that were at a moderate or low level in the fitness categories of each test. The programmes were based on World Health Organization (WHO) physical activity recommendations (12) and The American College of Sports Medicine (ACSM) general principles of exercise prescription regarding volume, i.e. intensity, frequency, duration of exercise activity.(13)

A total of 57 students were enrolled in the paramedic training program between 2012 and 2014 in Arcada UAS. Forty of these paramedic students participated in the baseline physical tests on a voluntary basis. Before the baseline tests the participants gave written consent to the health and physical activity data collected during the programme being used for scientific purposes. The ethical committee of the Hospital District of Helsinki and Uusimaa (HUS) has approved the study (HUS / 3574 / 2017). Subjects were sent an information letter explaining about the tests, about preparation 
before participating, and the test procedure. To enhance safe physical activity testing the subjects completed a standard pre-test health-screening questionnaire (13) and based on the results of this questionnaire none of the subjects had to be excluded. Thereafter the students participated in a physical fitness test protocol composed by the UKK institute (UKK Institute, Tampere, Finland).(14) Because the requirements of paramedics' clinical work are equal for both genders, the test criteria for male subjects were applied for both genders.

The tests were spread over two separate days on each occasion; the first morning for the body composition measurements and the second day for the physical performance tests. Basic demographic information about the subjects was recorded (age, gender, height, weight) at the first test session.

An InBody 720 Body Composition Analyzer (InBody Co., Ltd. Seoul. Korea) was used for measuring the subjects' body weight and body composition according to the manufacturer's guidelines. The inbuilt software was used to calculate body composition values such as body fat mass (FM), visceral fat (VF), skeletal muscle mass (SMM), relative FM of bodyweight, and body mass index (BMI; kg/m2). Bioelectrical impedance analysis has been proven to be a valid and reliable method for measurement of the body composition. $(15,16)$

The physical performance tests were conducted according to the following standardized procedure. The test session started with the WHO submaximal cycle ergometer test to estimate the subjects VO2Max, and was followed with a one-hour pause before the muscle tests. The muscle tests included were core muscle strength and stability test, push-ups, sit-ups, static back endurance test, isometric arm test, squats with extra weights, and grip strength. Tests were done in this particular order with a threeminute rest in between.

The submaximal cycle ergometer test protocol (17) was complemented by the non -exercise model.(18) The non-exercise model gives a prediction of the aerobic fitness for each subject, and gives an estimation of the workloads and heart rate levels during different stages at the test. Electronic ergo bikes (Daum electronic GmbH, Fürth, Germany) were used as cycle ergometers. The test started with a five-minute warm-up followed by 3-4 workloads of four minutes starting from a 50\% load of VO2Max and reaching an approximately load level of $80-85 \%$ of VO2Max. Pedalling frequency was kept between 55 and $75 \mathrm{rpm}$ by following the electronic bike's display, which shows the cadence continuously. Heart rate was monitored throughout the test with Polar T31 heart rate sensors (Polar Electro Ltd., Kempele, Finland).

The plank test designed by Brian Mackenzie was used to test global core muscle endurance. (19) This test consists of eight stages, starting from the plank exercise position supported by forearms and feet, and during the different stages arms and legs are raised. The test is timed, with a maximum time of 180 seconds.

Regarding the push-ups, the test was done according to the standard for males as mentioned in the American College of Sports Medicine (ACSM) exercise testing.(13) The maximum number of consecutive push-ups, without rest, was counted as the score.

Sit-ups were done in the supine position on a mat with the knees at 90 degrees and the arms placed with the fingers level with the ears, or interlocked behind the neck, the elbows touching the knees on rising. When going back down, and before starting a new sit-up, the shoulder blades must touch the mat. The tester gave light support from the ankles. The result was the number of sit-ups done in 60 seconds, without rest.

To measure static back muscle endurance, the subject was placed on the examining table with the lower body fixed to the table at the ankles and the upper body unsupported in a horizontal prone position. The arms were held alongside the body, but were not allowed to touch the table. The time, in seconds, in which the subject was able to keep the upper body straight and horizontal was recorded. The maximum time allowed for the test was 240 seconds.

An isometric arm test was used to measure the isometric strength and endurance of the arms and shoulders and the static strength of those body muscles supporting that movement. The subject stood in an upright position with the legs $15 \mathrm{~cm}$ apart. An $8 \mathrm{~kg}$ weight was held in the hands with the arms straight and horizontally level with the shoulders. The result was the time in seconds for which the subject was able to keep the weight steady at that horizontal level. The test was done in front of a mirror so that the 
subject could control the posture.

Squats with extra weights were used to test the muscular endurance of the legs. The subject stood in an upright posture with the feet $15 \mathrm{~cm}$ apart and with the feet slightly turned outward. For the test, the subject wore a vest for the extra weights, which were determined as $14 \mathrm{~kg}+1 / 3$ of the subject's own weight (e.g. if a person's weight is $72 \mathrm{~kg}$ 's, $1 / 3$ of the weight is $24 \mathrm{~kg}$ : the amount of extra weights is $14 \mathrm{~kg}+24 \mathrm{~kg}=38$ $\mathrm{kg}$ ). The subject had a 10-kg weight in each hand and the rest of the load was evenly distributed in the vest. From the upright starting position, the subject squatted so that the thighs were horizontal with the floor (knees bent at an angle of 90 degrees) and stood up from that position. A chair or step benches were used to mark the horizontal level. A maximum of 50 repetitions was performed.

Grip strength was measured with a hydraulic hand dynamometer (model SH5001, Saehan corporation, South Korea) with the subject seated, shoulder slightly abducted (the elbow is not allowed to touch the torso), elbow flexed to 90 degrees and the forearm in a neutral position.(20) Both arms were tested, with two trials for each arm with a 30 seconds rest between the trials. The best result was noted as the score in kilograms.

Statistical analyses were performed with SPSS (version 24.0; SPSS Inc., Chicago, Illinois, USA). Kolmogorov-Smirnov test was used to test data normality assumption, and variables without a normal distribution were analysed using a non-parametric test. Comparisons between the groups were performed with independent samples t-test, and comparisons within subjects (at follow-up - at baseline) with paired samples t-test (mean and its $95 \%$ confidence interval, CI). Effect sizes (ES) were calculated according to Cohen's $\mathrm{d}$ method. $\mathrm{P}<0.05$ (two-tailed) was accepted as a statistically significant threshold.

\section{Results}

Sixty-five percent $(26 / 40)$ of the students participated in the one-year follow-up examinations. Of the subjects, 14 were female, and 12 were male. The mean age of female subjects at baseline was 21.5 (standard deviation, SD, 1.8) years, and the corresponding age of males was 22.3 (SD 1.1) years. The mean follow-up time was one year. When the changes in anthropometrics during the follow-up period were analysed with female and male subjects combined, the mean weight and BMI (both p-values $<0.05$ ) slightly increased (Table 1). The baseline and follow-up characteristics and anthropometrics of the subjects are presented in Table 1.

At baseline male students had higher mean maximal oxygen consumption (VO2Max) than their female counterparts (mean difference $6.6 \mathrm{ml} / \mathrm{kg} / \mathrm{min}, \mathrm{p}<0.019$, Table 2). As expected, males had greater absolute muscle strength at baseline than females. On average the result of the core muscle strength and stability test $(\mathrm{p}=0.017)$, the push-up test $(\mathrm{p}<0.01)$, the isometric arm test $(\mathrm{p}<0.01)$, and grip strength of the right $(p<0.01)$ and left hand $(p<0.01)$ were greater in males than in females. Cardiorespiratory fitness and muscle strength results at baseline and at follow-up are presented in Table 2.

Overall, during the one-year follow-up the mean changes in muscle strength of the subjects were few. Females had improved their maximal oxygen consumption (VO2Max) on average by $2.4 \mathrm{ml} / \mathrm{kg} / \mathrm{min}$; ES 0.7. Female subjects had also improved their number of squats (ES 0.6), and males the number of push-ups (ES 1.1), and left arm grip strength (ES 1.0) (Table 2).

\section{Discussion}

This study reports over a one-year period the physical fitness of paramedic students who had participated in brief physical activity counselling with an individually tailored exercise programme. In some few muscle strength measurements the ESs were from moderate to large. Female students improved their maximal oxygen consumption and number of squats. Males improved number of push-ups, and left arm grip strength.

Fitness, gender, age, equipment, and demographic variables seem to be factors underlying to the high rate of paramedic occupational injuries. However, Jenkins et al. concluded, based on their review, that there is only little evidence available to quantify the relationship between pre-employment physical capacity testing and subsequent musculoskeletal injuries of paramedics. (21) In general, the evidence related to the effectiveness of pre-employment examinations targeted at specific occupational groups 
Table 1. Characteristics and anthropometrics of the study subjects at baseline, at follow-up and changes during the follow-up period.

\begin{tabular}{|c|c|c|c|c|c|c|c|c|c|}
\hline & \multicolumn{3}{|c|}{ Female $(N=14)$} & \multicolumn{3}{|c|}{ Male (N=12) } & \multicolumn{3}{|c|}{ All $(N=26)$} \\
\hline & Baseline & Follow-up & $\begin{array}{c}\text { Follow-up - } \\
\text { baseline }\end{array}$ & Baseline & Follow-up & Follow-up - baseline & Baseline & Follow-up & Follow-up - baseline \\
\hline $\begin{array}{l}\text { Characteristics and } \\
\text { anthropometrics }\end{array}$ & Mean $(\mathrm{SD})^{1}$ & Mean (SD) ${ }^{1}$ & $\begin{array}{l}\text { Mean change } \\
(95 \% \mathrm{CI})^{2}\end{array}$ & $\begin{array}{l}\text { Mean } \\
(\mathrm{SD})^{1}\end{array}$ & $\operatorname{Mean}(\mathrm{SD})^{1}$ & $\begin{array}{l}\text { Mean change } \\
(95 \% \mathrm{CI})^{2}\end{array}$ & Mean $(\mathrm{SD})^{1}$ & $\begin{array}{l}\text { Mean } \\
(\mathrm{SD})^{1}\end{array}$ & $\begin{array}{l}\text { Mean change } \\
(95 \% \mathrm{CI})^{2}\end{array}$ \\
\hline Age; yrs & $21.5(1.8)$ & $22.5(1.8)$ & & $22.3(1.1)$ & $23.3(1.1)$ & & $21.8(1.5)$ & $22.9(1.5)$ & \\
\hline Height; $\mathrm{cm}$ & $171.6(4.7)$ & & & $182.7(6.2)$ & & & $176.1(7.7)$ & & \\
\hline Weight; kg & $63.6(7.7)$ & $64.6(7.3)$ & $1.0(-0.33$ to 2.40$)$ & $78.0(10.0)$ & $79.6(10.9)$ & $1.6(-0.70$ to 3.93$)$ & $70.2(11.4)$ & $71.5(11.7)$ & $1.3(0.10 \text { to } 2.50)^{*}$ \\
\hline $\mathrm{BMI}^{3} ; \mathrm{kg} / \mathrm{m}^{2}$ & $21.8(3.0)$ & $22.2(2.8)$ & $0.4(-0.09$ to 0.87$)$ & $23.5(2.5)$ & $24.0(2.7)$ & $0.5(-0.21$ to 1.28$)$ & $22.6(2.9)$ & $23.0(2.9)$ & $0.5(0.06 \text { to } 0.86)^{*}$ \\
\hline Lean mass; $\mathrm{kg}$ & $27.5(2.7)$ & $27.8(2.3)$ & $0.3(-0.23$ to 0.86$)$ & $38.6(4.9)$ & $39.0(4.8)$ & $0.4(-0.41$ to 1.13$)$ & $32.6(6.8)$ & $33.0(6.7)$ & $0.3(-0.09$ to 0.76$)$ \\
\hline Fat mass; $\mathrm{kg}$ & $14.0(5.3)$ & $14.6(5.3)$ & $0.6(-0.38$ to 1.61$)$ & $10.4(3.5)$ & $11.5(4.8)$ & $1.1(-1.03$ to 3.20$)$ & $12.4(4.8)$ & $13.2(5.2)$ & $0.8(-0.20$ to 1.86$)$ \\
\hline
\end{tabular}

${ }^{1}$ SD: Standard deviation

${ }^{2} \mathrm{CI}$ : Confidence interval

${ }^{3}$ BMI: Body mass index

$* \mathrm{p}<0.05$

Table 2. Cardiorespiratory fitness, and muscle strength of the female andmale subjects at baseline, at follow-up and changes during the follow-up period

\begin{tabular}{|c|c|c|c|c|c|c|c|c|c|}
\hline \multirow[b]{3}{*}{ Test } & \multicolumn{3}{|c|}{ Female $(\mathrm{N}=14)$} & \multicolumn{3}{|c|}{ Male $(\mathrm{N}=12)$} & \multicolumn{3}{|c|}{ All $(\mathrm{N}=26)$} \\
\hline & Baseline & Follow-up & Follow-up - baseline & Baseline & Follow-up & Follow-up - baseline & Baseline & Follow-up & Follow-up - baseline \\
\hline & Mean $(\mathrm{SD})^{1}$ & Mean $(\mathrm{SD})^{1}$ & Mean change $(95 \% \mathrm{CI})^{2}$ & $\operatorname{Mean}(\mathrm{SD})^{1}$ & Mean $(\mathrm{SD})^{1}$ & Mean change $(95 \% \mathrm{CI})^{2}$ & $\operatorname{Mean}(\mathrm{SD})^{1}$ & $\operatorname{Mean}(\mathrm{SD})^{1}$ & Mean change $(95 \% \mathrm{Cl})^{2}$ \\
\hline $\mathrm{VO}_{2 \mathrm{Max}} ; \mathrm{ml} / \mathrm{kg} / \mathrm{min}$ & $39.8(5.6)$ & $42.3(5.4)$ & $2.4(0.09$ to 4.74$) *$ & $45.9(7.4)$ & $47.3(8.2)$ & $1.4(-1.98$ to 4.82$)$ & $42.9(7.1)$ & $44.8(7.3)$ & $1.9(0.01$ to 3.82$) *$ \\
\hline $\begin{array}{l}\text { Core muscle strength and } \\
\text { stability test; } s\end{array}$ & $139.6(33.0)$ & $149.6(36.8)$ & $10.0(-0.19$ to 20.19$)$ & $165.8(19.6)$ & $171.3(20.7)$ & $5.4(-6.56$ to 17.40$)$ & $152.7(29.7)$ & $160.4(31.2)$ & $7.7(0.41 \text { to } 15.00)^{* *}$ \\
\hline Push-up; $\mathrm{n}^{3}$ & $11.7(6.8)$ & $12.8(6.5)$ & $1.1(-2.69$ to 4.87$)$ & $29.3(10.3)$ & $34.0(8.6)$ & $4.8(1.93 \text { to } 7.57)^{*}$ & $20.9(12.4)$ & $23.9(13.2)$ & $3.0(0.72 \text { to } 5.28)^{*}$ \\
\hline Sit-up; $n^{3}$ & $30.7(7.8)$ & $30.6(4.6)$ & $-0.1(-3.75$ to 3.59$)$ & $33.8(7.5)$ & $35.0(9.2)$ & $1.2(-1.67$ to 4.01$)$ & $32.3(7.7)$ & $32.8(7.5)$ & $0.5(-1.61$ to 2.69$)$ \\
\hline Static back test; $s$ & $186.2(54.7)$ & $184.3(52.0)$ & $-1.8(-30.26$ to 26.60$)$ & $167.1(47.2)$ & $151.5(29.5)$ & $-15.5(-44.17$ to 13.08$)$ & $177.0(51.0)$ & $168.7(45.0)$ & $-8.4(-27.12$ to 10.34$)$ \\
\hline Isometric arm test; $\mathrm{n}^{3}$ & $27.9(12.8)$ & $28.9(10.0)$ & $1.0(-3.36$ to 5.36$)$ & $60.5(14.6)$ & $58.9(11.5)$ & $-1.6(-8.97$ to 5.72$)$ & $44.2(21.4)$ & $43.9(18.6)$ & $-0.3(-4.28$ to 3.66$)$ \\
\hline Squats; $\mathrm{n}^{3}$ & $39.3(14.2)$ & $43.5(9.7)$ & $4.2(0.01 \text { to } 8.33)^{*}$ & $45.7(7.5)$ & $41.5(11.1)$ & $-4.3(-11.01$ to 2.47$)$ & $42.4(11.7)$ & $42.5(11.7)$ & $0.1(-3.87$ to 4.13$)$ \\
\hline Grip strength, $\mathrm{dx} ; \mathrm{kg}$ & $38.7(7.2)$ & $38.5(7.1)$ & $-0.2(-3.04$ to 2.71$)$ & $55.8(8.4)$ & $58.1(7.6)$ & $2.3(-0.81$ to 5.47$)$ & $47.2(11.6)$ & $48.3(12.3)$ & $1.1(-0.95$ to 3.11$)$ \\
\hline Grip strength, sin; $\mathrm{kg}$ & $35.3(5.8)$ & $36.8(5.1)$ & $1.6(-1.35$ to 4.52$)$ & $52.6(8.4)$ & $56.6(7.3)$ & $4.0(1.39 \text { to } 6.53)^{*}$ & $43.9(11.4)$ & $46.7(11.8)$ & $2.8(0.91$ to 4.64$) *$ \\
\hline
\end{tabular}

${ }^{1}$ SD: Standard deviation

${ }^{2} \mathrm{CI}$ : Confidence interval

${ }^{3} \mathrm{n}$ : Number of repetitions

${ }^{*} \mathrm{p}<0.05$

$* * \mathrm{p}=0.05$, non-parametric test

or health risks in reducing occupational disease, injury or sick leave is inconsistent.(22)

Videman et al. investigated back pain among nursing students with a 7.5-year follow-up. Prevalence of back pain increased sharply during nursing school but thereafter more slowly. Back pain when starting nursing studies was a predictor for back-related pain and disability at follow-up. Self-reported work-related physical workload was associated with back pain and related disability.(23) Furthermore, Lallukka et al. showed that early exposure to heavy physical workload is associated with a high risk of radiating low back pain after a follow-up of 21 years. The risks were particularly pronounced and robust among women. Therefore care should be taken when young people enter the labour market and are exposed to physically loading work (24) as occasionally is the case among paramedics.

One limitation of our study was that we did not systematically evaluate the volume of physical activity during follow-up. However, at the follow-up examination $92 \%$ of the subjects reported that they had engaged regularly in leisure-time exercise for one month prior to the follow-up examination.

It seems to be possible to maintain, and even slightly improve the physical condition of paramedic students with a self-directed, guided exercise programme.(25) Educational institutes providing education and training for paramedics need to find new and more effective ways of encouraging students adopt living habits conducive to good physical condition. 


\section{Acknowledgments}

The authors wish to thank all the paramedic students of Arcada University of Applied Sciences who contributed to the study. We also wish to thank Mrs. Virginia Mattila, for the final correction of the language of the article.

\section{References}

1. Sterud T, Ekeberg, Ø, Hem, E. Health status in the ambulance services: a systematic review. BMC Health Services Research. 2006;6: 82.

2. Maguire B, Hunting K, Guidotti T, Smith G. Occupational injuries among emergency medical services personnel. Prehosp Emerg Care. 2005; 9: 405-11.

3. Reichard A, Jackson L. Occupational injuries among emergency responders. Am J Ind Med 2010; 53 (1): 1-11.

4. Thornton K, Sayers M. Unfit for duty? Evaluation of 4 years of paramedic preemployment fitness screening test results. Prehospital Emergency Care 2014; 18 (2): 201-206.

5. Aasa U, Barnekow-Bergkvist M, Änquist K-A, Brulin C. Relationship between work-related factors and disorders in the neck-shoulder and low-back region among female and male ambulance personnel. J Occup Health. 2005; 47: 481-9.

6. Gamble R, Stevens A, McBrien H, Black A, Cran G, Boreham C. Physical fitness and occupational demands of the Belfast ambulance service. Br J Ind Med. 1991; 48: 592-6.

7. Pattani S, Constantinovici N, Williams S. Who retires early from the NHS because of ill health and what does it cost? A national cross sectional study. BMJ. 2001; 322: 208-9.

8. Crill M, Hostler D. Back strength and flexibility of EMS providers in practicing prehospital providers. Journal of Occupational Rehabilitation. 2005; 15(2): 105111.

9. Laska M, Pasch K, Lust K, Story M., Ehlinger, E. The Differential Prevalence of Obesity and Related Behaviors in Two vs. Four-Year Colleges. Obesity 2011; 19 (2): 453-6.

10. Schluter P, Turner C, HuntingtonA, Bain C, McClure R. Work/life balance and health: the nurses and midwives e-cohort study. International Nursing Review 2011; 58: 28-36.

11. Chapman D, Peiffer J, Abbiss C, Laursen P. A descriptive profile of Western Australian male paramedics. Journal of Emergency Primary Health Care 2007; 5 (1).

12. WHO. Global recommendations on physical activity for health. World Health Organization 2010. ISBN 9789241599979.

13. The American College of Sports Medicine (ACSM) exercise testing. ACSM' s Guidelines for Exercise Testing and Prescription, 8th Edition. American College of Sports Medicine. Philadelphia: Lippincott Williams \& Wilkins. 2009. pp.

14. http://www.ukkinstituutti.fi/filebank/500-ALPHAFIT Testers Manual.pdf. Accessed 31.5.2017.

15. Salmi Jukka. Body Composition Assessment with segmental multifrequency bioimpedance method. Journal of Sports Science \& Medicine 2003; 2. Supplementum 3 .

16. Aandstad A, Holtberget K, Hageberg, R, Holme I, Anderssen Sigmund. Validity and Reliability of Bioelectrical Impedance Analysis and Skinfold Thickness in Predicting Body Fat in Military Personnel. Military Medicine 2014; 179 (2): p. 208-217.

17. Lange Andersen K, Shephard R, Denolin H, Varnauskas E, Masironi R. 1971. Fundamentals of exercise testing. Geneva: WHO.

18. Jackson Andrew, Blai Steven, Mahar Matthew, Wier Larry, Ross Robert, Stuteville Joseph. Prediction of functional aerobic capacity without exercise testing. Med. Sci. Sports Exerc. 1990. 22; (6): pp. 863-870.

19. Mackenzie B. 101 Performance Evaluation Tests. Jonathan Pye. Electric World plc. London; 2005. p. 111-2.

20. Fess E. Grip Strength. Clinical Assessment Recommendations. In: Casanova J. (Ed.) Grip Strength. American Society of Hand Therapists. Chicago. 1992. pp.41- 
5 .

21. Jenkins N, Smith G, Stewart S, Kamphuis C. Pre-employment physical capacity testing as a predictor for musculoskeletal injuries in paramedics: a review of the literature. Work. 2016; 55: 565-75.

22. Schaafsma F, Mahmud N, Reneman N, Fassier J, Jungbauer F. Pre-employment examinations for preventing injury, disease and sick-leave in workers. Cochrane Database of Systematic Reviews 2016; (1) Art. No.:CD008881. DOI.10.1002/14651858.CD00888.1.pub2.

23. Videman T, Ojajärvi A, Riihimäki H, Troup J. Low back pain among nurses. A follow-up beginning at entry to the nursing school. Spine. 2005; 30 (20): 23342341 .

24. Lallukka T, Viikari-Juntura E, Viikari J, Kähönen M, Lehtimäki T, Raitakari OT, Solovieva S. Early work-related physical exposures and low back pain in midlife: the Cardiovascular Risk in Young Finns Study. Occup Environ Med. 2017 (74): 163-168.

25. Aasa A, Angquist K, Barnekow-Bergkvist M. The effects of a 1-year physical exercise programme on development of fatigue during a simulated ambulance work task. Ergonomics 2008; 51(8): 1179-1194. 